\title{
Boundary Values of Degenerate Configuration Core of White Dwarfs using Ramanujan's Method
}

\author{
A. M. Prince ${ }^{1 *}$, S. Thomas ${ }^{1}$, S. George ${ }^{2}$ \\ ${ }^{1}$ Department of Physics, SHUATS, Prayagraj, India \\ ${ }^{2}$ Department of Mathematics and Statistics, SHUATS, Prayagraj, India
}

Received 2 August 2019, accepted in final revised form 25 September 2019

\begin{abstract}
In this paper we have found out boundary values of degenerate configuration core of White Dwarf using Ramanujan's method. By converting non-linear Lane Emden-type equation for degenerate core into a series using an iterative method. Considering the first five terms of the series numerical values has been calculated. The applied method is efficient and simplifies the calculation. The obtained result has been compared with exact values. Ramanujan's method and the new iterative method are used for the first time to solve the Lane Emden equation for white dwarf core.
\end{abstract}

Keywords: Lane Emden-type Equation; Iterative method; Ramanujan's method.

(c) 2020 JSR Publications. ISSN: 2070-0237 (Print); 2070-0245 (Online). All rights reserved. doi: http://dx.doi.org/10.3329/jsr.v12i1.42454 J. Sci. Res. 12 (1), 69-74 (2020)

\section{Introduction}

Star, always a source of wonder as well as science is the most common existent we find in a galaxy and white dwarf, the later evolutionary stage of a star holds the second position. Stars which have masses less than $8 \mathrm{M}_{\text {sun }}$ will end up in this form. The first white dwarf identified is Sirius B. White dwarfs differ from other stars in some fundamental way, firstly they are much fainter as compared to an average star of same mass, as all helium and hydrogen inside the core is converted to oxygen and carbon and no further fusion takes place because of its lower initial mass thereby prevents the generation of internal pressure. Instead of energy generation the gradual emission of large amount of stored heat is the reason for the luminosity of white dwarfs, as it moves on HR diagram.

Secondly the degenerate nature of electron gas in the interior of white dwarfs, as they don't follow perfect gas equation [1]. The extreme outer layers of white dwarf contains non degenerate matter which follows the gas law, $\mathrm{P} \propto \mathrm{T}$. The core of white dwarf is highly dense and electrons are packed tightly and all the energy levels from ground level up are filled obeying Pauli's Exclusion Principle and electron gas attains the status 'degenerate gas'. Degenerate configurations follow the relation $\mathrm{P}=\mathrm{K}_{1} \rho^{5 / 3}$ in non-relativistic conditions

\footnotetext{
${ }^{*}$ Corresponding author: achumary29@gmail.com
} 
which represent Lane Emden polytropes with index $n=3 / 2$. As the mass of white dwarf increases, the internal pressure increases in turn the velocity of electrons approach speed of light making the special relativity accountable and pressure density relation takes the form $\mathrm{P}=\mathrm{K}_{2} \rho^{4 / 3}$ and are polytropes of index $\mathrm{n}=3$ [2].

Thirdly in contradiction, more massive stars are smaller and also there is a limiting mass where degeneracy pressure exists without collapse, which is termed as Chandrasekhar limit [3]. The inversely proportional mass -radius relation of a white dwarf is described as $R \propto M^{-1 / 3}$ and limiting mass is $1.4 \mathrm{M}_{\text {sun }}$ [4].

\section{White Dwarf Equation}

Idealising white dwarf as complete degenerate configuration and neglecting the gaseous fringe and moving towards the model equation for it. The second order structural equation of degenerate core in differential form is,

$$
\frac{1}{\eta^{2}} \frac{d}{d \eta}\left(\eta^{2} \frac{d \phi}{d \eta}\right)=-\left(\phi^{2}-\frac{1}{y_{0}^{2}}\right)^{3 / 2}
$$

with boundary conditions, $\phi(0)=1, \phi\left(\eta_{1}\right)=\frac{1}{y_{0}}$ such that $\phi$ takes the value unity at origin and $\eta_{1}$ specifies the boundary where the density vanishes. $\phi$-density of white dwarf, $\eta$ - dimensionless radial distance from the centre and $\frac{1}{y_{0}^{2}}$ is a constant related to the density of white dwarf at the centre.

Our goal is to investigate a solution for equation (1). Different numerical methods were employed for the solution. General first kind of Lane Emden equation was solved by many researchers [5-14].

The special case of white dwarf equation was studied by Hojjati and Parand [15], Cardenas [16], Das, Ghosh et al. [17], Nouh [18], Singh [19], Aydinlink and Kiris [20].

In this paper we used new iterative method for solving the white dwarf equation (1) and the roots of the equation for different values of $\frac{1}{y_{0}^{2}}$ is obtained using Ramanujan's method.

\section{New Iterative Method}

Consider the functional equation

$$
t(x)=N(t)+f(x)
$$

Where $\mathrm{N}$ is the nonlinear operator from a Banach space $\mathrm{B} \rightarrow \mathrm{B}$ and $f(x)$ is a known function.

The solution of equation (2) is assumed to be in the series form [21]

$$
t=\sum_{i=0}^{\infty} t_{i}
$$

The nonlinear operator can be decomposed as 


$$
N\left(\sum_{i=0}^{\infty} t_{i}\right)=N\left(t_{0}\right)+\sum_{i=1}^{\infty}\left[N\left(\sum_{j=0}^{i} t_{j}\right)-N\left(\sum_{j=0}^{i-1} t_{j}\right)\right]
$$

So equation (2) can be re-written as

$$
\sum_{i=0}^{\infty} t_{i}=f+N\left(t_{0}\right)+\sum_{i=1}^{\infty}\left[N\left(\sum_{j=0}^{i} t_{j}\right)-N\left(\sum_{j=0}^{i-1} t_{j}\right)\right]
$$

We define the recurrence relation

$$
\begin{aligned}
& t_{0}=f \\
& t_{1}=N\left(t_{0}\right) \\
& t_{2}=N\left(t_{0}+t_{1}\right)-N\left(t_{0}\right) \\
& t_{m+1}=N\left(t_{0}+\cdots+t_{m}\right)-N\left(t_{0}+\cdots+t_{m-1}\right) m=1,2 \ldots . .
\end{aligned}
$$

then

$$
\left(t_{1}+\cdots+t_{m+1}\right)=N\left(t_{0}+\cdots+t_{m}\right) \quad m=1,2 \cdots
$$

and

$$
t=f+\sum_{i=1}^{\infty} t_{i}
$$

If $\mathrm{N}$ is a contraction, that is ||$N(x)-N(t)|| \leq K|| x-t||, 0<K<1$, then

$$
\begin{gathered}
|| t_{m+1} \|=|| N\left(t_{0}+\cdots+t_{m}\right)-N\left(t_{0}+\cdots+t_{m-1}\right)|| \leq K|| t_{m}|| \leq K^{m}|| t_{0}|| \\
m=0,1,2 \cdots
\end{gathered}
$$

And the series $\sum_{i=1}^{\infty} t_{i}$ absolutely and uniformly converges to a solution of equation (2) [22] which is unique in view of Banach fixed point theorem [23].

\section{Ramanujan's Method}

The smallest root of the equation

$f(x)=0$

where $\mathrm{f}(\mathrm{x})$ is of the form $f(x)=1-\left(a_{1} x+a_{2} x^{2}+a_{3} x^{3}+\cdots.\right)$ using an iterative method is described [24,25].

For smaller values of $\mathrm{x}$, we can write

$$
\left[1-\left(a_{1} x+a_{2} x^{2}+a_{3} x^{3}+\cdots .\right)\right]^{-1}=b_{1}+b_{2} x+b_{3} x^{2}+\cdots
$$

Expanding left hand side by Binomial theorem and comparing the coefficients of like powers of $\mathrm{x}$ on both sides we get

$$
\begin{aligned}
& b_{1}=1 \\
& b_{2}=a_{1}=a_{1} b_{1} \\
& b_{3}=a_{1}^{2}+a_{2}=a_{1} b_{2}+a_{2} b_{1} \\
& b_{n}=a_{1} b_{n-1}+a_{2} b_{n-2}+\cdots+a_{n-1} b_{1} \quad n=2,3, . .
\end{aligned}
$$

The successive convergents, viz., $b_{n} / b_{n+1}$ approach root of the equation (4) of the above given form. 


\section{Solution of White Dwarf Equation}

The Volterra integral form of first kind of Lane Emden equation of the form,

$$
\begin{aligned}
& \phi^{\prime \prime}+\frac{2}{\eta} \phi^{\prime}+(\phi)^{m}=0 ; \phi(0)=1 \quad \phi^{\prime}(0)=0 \text { is set as [26]. } \\
& \phi(\eta)=1-\int_{0}^{\eta} t\left(1-\frac{t}{\eta}\right) \phi^{m} d t
\end{aligned}
$$

Differentiating the equation, using Leibnitz rule

$$
\phi^{\prime}(\eta)=-\int_{0}^{\eta}\left(\frac{t^{2}}{\eta^{2}}\right) \phi^{m} d t
$$

Solving equation (4) using New Iterative Method

$$
\phi(\eta)=-\int_{0}^{\eta} \int_{0}^{\eta}\left(\frac{t^{2}}{\eta^{2}}\right) \phi^{m} d t d \eta
$$

White dwarf equation can be written in the form

$$
\phi^{\prime \prime}+\frac{2}{\eta} \phi^{\prime}+\left(\phi^{2}-\frac{1}{y_{0}^{2}}\right)^{3 / 2}=0
$$

Here $\phi^{m}$ takes the form $\left(\phi^{2}-\frac{1}{y_{0}^{2}}\right)^{3 / 2}$ where $\frac{1}{y_{0}^{2}}$ takes value from 0 to 0.9

$$
\left(\phi^{2}-\frac{1}{y_{0}^{2}}\right)^{3 / 2} \approx q^{3}+3 q(\phi-1)+\frac{3 q^{2}+3}{2 q}(\phi-1)^{2}+\frac{9 q^{2}-3}{3 ! q^{3}}(\phi-1)^{3}+\cdots
$$

Where $q^{2}=1-\frac{1}{y_{0}^{2}}$

Substituting the expansion in equation (6) and using the recurrence relation given in (3)

$$
\begin{aligned}
& \phi_{0}=1 \\
& \phi_{1}=\mathrm{N}\left(\phi_{0}\right)=-\int_{0}^{\eta} \int_{0}^{\eta} \frac{\mathrm{t}^{2}}{\eta^{2}} \phi_{0} \mathrm{dtd} \eta=-\frac{\eta^{2}}{6} \mathrm{q}^{3} \\
& \phi_{2}=\mathrm{N}\left(\phi_{0}+\phi_{1}\right)-\mathrm{N}\left(\phi_{0}\right)=-\int_{0}^{\eta} \int_{0}^{\eta} \frac{\mathrm{t}^{2}}{\eta^{2}}\left(\phi_{0}+\phi_{1}\right) \mathrm{dtd} \eta+\int_{0}^{\eta} \int_{0}^{\eta} \frac{\mathrm{t}^{2}}{\eta^{2}} \phi_{0} \mathrm{dtd} \eta=\frac{\eta^{4}}{40} \mathrm{q}^{4} \\
& \phi_{3}=\mathrm{N}\left(\phi_{0}+\phi_{1}+\phi_{3}\right)-\mathrm{N}\left(\phi_{0}+\phi_{1}\right)=-\frac{\eta^{6}}{7 !} \mathrm{q}^{5}\left(\left(5 \mathrm{q}^{2}+14\right)\right.
\end{aligned}
$$

Summing up the series

$$
\phi(\eta)=\phi_{0}+\phi_{1}+\phi_{2}+\cdots=1-\frac{\eta^{2}}{6} q^{3}+\frac{\eta^{4}}{40} q^{4}-\frac{\eta^{6}}{7 !} q^{5}\left(\left(5 q^{2}+14\right)+\cdots\right.
$$

which is the series solution of white dwarf structure equation. The series is solved using Ramanujan's method.

\section{Results and Discussion}

The obtained $\eta_{1}$ for different values of $\frac{1}{y_{0}^{2}}$ using Ramanujan's method is presented in Table 1. The second column gives the boundary value for polytropic index $n=3$ found using Ramanujan's method while third column gives Chandrasekhar's values [27]. The fourth column gives the percentage error of the boundary values. The method employed gives result which almost matches with the earlier obtained results. The percentage error $\sigma$ is $1 \%$ which shows the efficiency of Ramanujan's method in solving such equations. 
Table 1. The comparison for boundary value $\left(\eta_{1}\right)$ using Ramanujan's method with Chandrasekhar's value.

\begin{tabular}{llll}
\hline $1 / \mathrm{y}_{0}{ }^{2}$ & $\begin{array}{l}\text { Ramanujan } \\
\text { method }\left(\eta_{1}\right)\end{array}$ & $\begin{array}{l}\text { Chandrasekhar's } \\
\text { value }\left(\eta_{1}\right)\end{array}$ & \% error \\
\hline 0.00 & 7.17137 & 6.8968 & 3.9811 \\
0.01 & 5.36011 & 5.3571 & 0.0563 \\
0.02 & 4.98109 & 4.9857 & 0.0925 \\
0.05 & 4.45916 & 4.4601 & 0.0212 \\
0.10 & 4.07569 & 4.0690 & 0.1646 \\
0.20 & 3.74124 & 3.7271 & 0.3793 \\
0.30 & 3.59787 & 3.5803 & 0.4907 \\
0.40 & 3.54415 & 3.5245 & 0.5575 \\
0.50 & 3.55423 & 3.5330 & 0.6008 \\
0.60 & 3.62650 & 3.6038 & 0.6300 \\
0.80 & 4.07155 & 4.0446 & 0.6663 \\
\hline
\end{tabular}

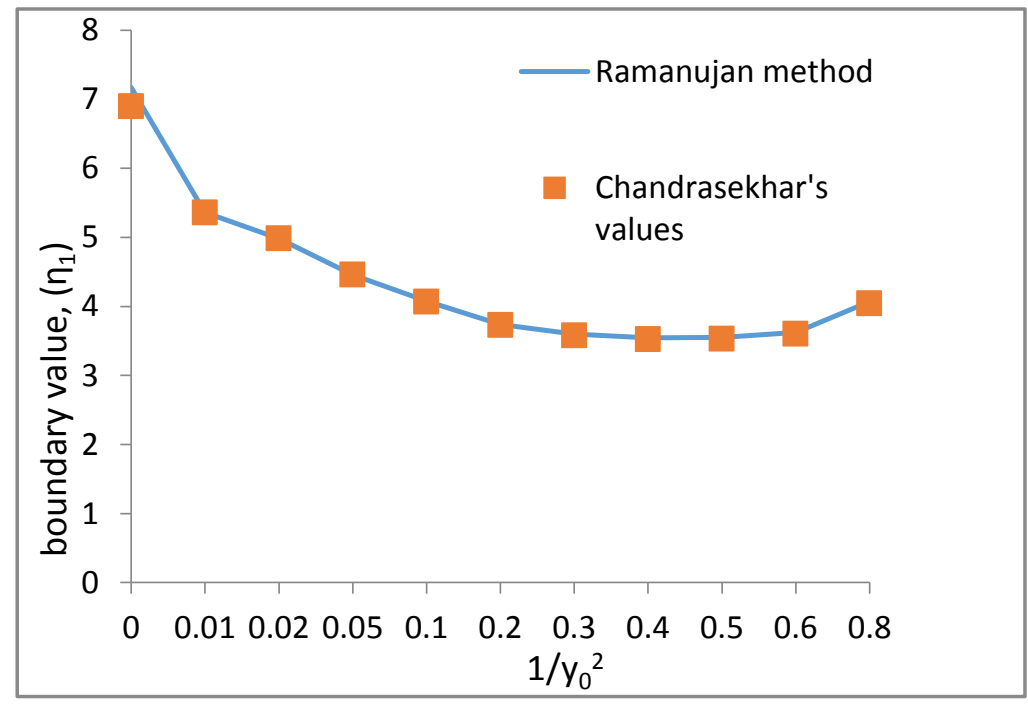

Fig. 1. Comparison between numerical values obtained using Ramanujan's method with Chandrasekhar's values.

\section{Conclusion}

The purpose of this paper was to suggest a numerical method for solving structural equation of degenerate core of white dwarf. The method is based on converting nonlinear differential equation to integral form [7] and obtains a series solution using New Iterative Method [21], which is solved using Ramanujan's method. The Fig. 1 and Table 1 compares the numerical result obtained by the suggested method and the results given by Chandrasekhar [27] which clearly shows the efficiency of the method. New iterative 
method used for series finding and the Ramanujan's method used for obtaining boundary values (root of the series) is the unique identity of this paper

\section{References}

1. R. H. Fowler, Monthly Notices 87, 114 (1926). https://doi.org/10.1093/mnras/87.2.114

2. S. Chandrasekhar, Philosophical Magazine 11, 592 (1931). https://doi.org/10.1080/14786443109461710

3. S. Chandrasekhar, Astrophysical J. 74, 81 (1931). https://doi.org/10.1086/143324

4. S. Chandrasekhar, Monthly Notices 95, 207 (1935). https://doi.org/10.1093/mnras/95.3.207

5. P. Pascual, Astron.Astrophys, 60, 161 (1977).

6. C. Mohan and A. R. Al-Bayaty, Astrophys. Space Sci. 73, 227 (1980). https://doi.org/10.1007/BF00642378

7. A. M. Wazwaz, Appl. Math. Comput. 118, 287 (2001). https://doi.org/10.1016/S0096-3003(99)00223-4

8. J. H. He, Appl. Math. Comput. 143, 539 (2003). https://doi.org/10.1016/S0096-3003(02)00382-X

9. S. A. Yousefi, Appl. Math. Comput. 175, 574 (2006). https://doi.org/10.1016/j.amc.2005.07.032

10. A. Yildirim, T. Ozis, Phys. Letter A 369, 70 (2007). https://doi.org/10.1016/j.physleta.2007.04.072

11. M. Dehghan and F.Shakeri, New. Astron. 13, 53 (2008). https://doi.org/10.1016/j.newast.2007.06.012

12. T. Tanriverdi, British J. Math. Comput. Sci. 20, 1 (2017)

13. K. Parand and S. Hashemi, Ain Shams Eng. J. 9, 615 (2018). https://doi.org/10.1016/j.asej.2016.03.010

14. P. Maheshwar and P. Joshi, Astrophysics Space Sci. 363, 9 (2018).

15. G. Hojjati and K. Parand, World Acd. Sci. Eng. Technol. 56, 889 (2011).

16. P. P. Cardenas and A. U. William, Appl. Math. 4, 28 (2014). https://doi.org/10.1016/j.aml.2014.01.006

17. D. Das, D. Ghosh, B. K. Datta, R. K. Bera, P. K. Sahu, and S. S. Roy, Int. J. Appl. Comput. Math. 1, 369 (2015). https://doi.org/10.1007/s40819-014-0020-8

18. M. I. Nouh, A. -B. Abdel-Salam, Emad, eprint arXiv:1705.03749 (2017)

19. H. Singh, Astrophy. Space Sci 363, 71 (2018). https://doi.org/10.1007/s10509-018-3286-1

20. S. Aydinlik and A. Kiris, Astrophy. Space Sci. 363, 71 (2018). https://doi.org/10.1007/s10509018-3483-y

21. V. Daftardar and H. Jafari, J. Math. Anal. Appl. 316, 753 (2006). https://doi.org/10.1016/j.jmaa.2005.05.009

22. Y. Cherrault, Kybernetes 8, 31 (1988). https://doi.org/10.1108/eb005812

23. A. J. Jerri, Introduction to Integral Equation with Applications, $2^{\text {nd }}$ Edition (Wiley Interscience, New York, 1999).

24. S. S. Shastri, Introductory Methods to Numerical Analysis (Prentice Hall of India, 2008).

25. B. C. Berndt, Ramanujan's Notebooks-Part I (Springer, Switzerland, 1985). https://doi.org/10.1007/978-1-4612-1088-7

26. M. A. Al- Jawary and H. R. Al-Qaissy, Monthly Notices 448, 3093 (2015). https://doi.org/10.1093/mnras/stv198

27. S. Chandrasekhar, An Introduction to the Study of Stellar Structure (Dover Publications, New York, USA, 1939). 